\title{
Harmonic Domain Dynamic Transfer Function of a Nonlinear Time-Periodic Network
}

\author{
Taku Noda, Member, IEEE, Adam Semlyen, Life Fellow, IEEE, and Reza Iravani, Senior Member, IEEE
}

\begin{abstract}
This paper presents a new concept called harmonic domain dynamic transfer function (HDDTF), which characterizes the dynamics of a nonlinear, time-periodic network as seen from a port (or multiple ports), in terms of the frequency response of harmonic perturbations superimposed on its underlying periodic steady state. It pertains to the transient behavior superimposed on the steady state. The HDDTF is a transfer-function matrix $\boldsymbol{H}(s)$ relating the vectors of harmonic domain input and output endowed with $s$-domain properties. Because the network can contain saturable (nonlinear) elements and periodically-switching (time-periodic) power electronics components, the HDDTF may be used for the analysis of power quality problems. It may also serve for the identification of a reduced-order dynamic equivalent of a nonlinear, time-periodic network to be used in time-domain transient simulations. The HDDTF is obtained by linearization, about the periodic steady state, of the nonlinear state equations describing a given network. Following the derivation of the HDDTF, a modal analysis to characterize the HDDTF by its diagonalization is presented. Two test systems are used to produce numerical examples.
\end{abstract}

Index Terms-Dynamics, electromagnetic transient analysis, large-scale systems, nonlinear circuits, periodic functions, power electronics, power quality, power system harmonics, saturable cores, transfer functions.

\section{INTRODUCTION}

$\mathbf{H}$ ARMONIC sources have a primordial role in the operation of interconnected power systems and lead to important power quality assessment issues [1], [2]. Such sources are usually nonlinear or time-periodic system components; the nonlinear components include saturable elements such as the magnetizing circuits of transformers, and the time-periodic elements include periodically-switching power electronics converters. It is often useful to characterize the dynamics of a network in a compact form, and transfer functions like impedance and admittance matrices are used to this end for linear time-invariant networks. However, if the network is nonlinear and time-periodic, such an entity is, to our knowledge, not available at this time.

In this paper, a concept called harmonic domain dynamic transfer function (HDDTF) is introduced. The HDDTF characterizes the dynamics of a nonlinear and time-periodic network,

Manuscript received February 17, 2002. This work was supported by the Natural Sciences and Engineering Research Council (NSERC) of Canada.

T. Noda is with Electrical Insulation Department, Central Research Institute of Electric Power Industry, Tokyo 201-8511, Japan (e-mail: takunoda@ieee.org). He is currently a Visiting Scientist at the University of Toronto, ON, Canada.

A. Semlyen and R. Iravani are with the Department of Electrical and Computer Engineering, University of Toronto, Toronto, ON, M5S 3G4, Canada (e-mail: adam.semlyen@utoronto.ca; iravani@ecf.utoronto.ca).

Digital Object Identifier 10.1109/TPWRD.2003.817788 operating in its periodic steady state, in terms of the frequency response of perturbations of harmonic components. The network is seen from a port or multiple ports, and the nonlinear state equations that describe the given network are linearized about the periodic steady state. The HDDTF is then calculated from the linearized state equations with periodic coefficients. If the port voltages and currents are selected as the input and the output of the state equations, respectively, such an HDDTF may be called a harmonic domain dynamic admittance matrix (HDDAM), and it is compatible with admittance- and conductance-based methods widely used in power system analysis. The concept of the HDDTF can be considered as an extension of its static case, which is used for steady-state analysis [3], [4]. The HDDTF includes the information not only of steady state but also of the frequency response of harmonic perturbations in addition to the static case. Thus, equivalently, the HDDTF characterizes the transient behavior superimposed on the periodic steady state in the time domain. In short, the HDDTF is a transfer-function matrix $H(s)$ relating the vectors of harmonic domain input and output endowed with $s$-domain properties. This paper also presents a modal decomposition analysis to characterize the HDDTF by its diagonalization. The modal domain equations give simpler and more tangible interpretation of the HDDTF.

In HDDTF applications, the network can contain saturable (nonlinear) elements and periodically-switching (time-periodic) power electronics components. Therefore, the HDDTF may be used for the analysis of power quality problems. It can also be used for the identification of a reduced-order dynamic equivalent of a nonlinear, time-periodic network for time domain transient simulations as presented by the authors [5]. In this paper, a simple illustrative network and a relatively large network are used to provide numerical examples.

\section{DERIVATION OF HDDTF}

\section{A. Nonlinear Time-Periodic Network}

Let us consider a nonlinear time-periodic network, operating in periodic steady state with the base frequency $f_{0}$, as seen from a port or from multiple ports as shown in Fig. 1. The number of state variables in the network is $N$, and the number of terminals (phases) in the port(s) is $N_{p h}$. The network consists of linear time-invariant $R, L, C$ components, nonlinear $R, L, C$ components, periodically operating switches, and independent voltage and current sources with periodic waveforms. The operating period of the switches and the sources is $T\left(=1 / f_{0}=2 \pi / \omega_{0}\right)$, and therefore, the waveforms of all voltages and currents are $T$-periodic. To obtain the underlying periodic steady state, the 


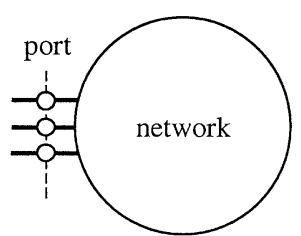

(a)

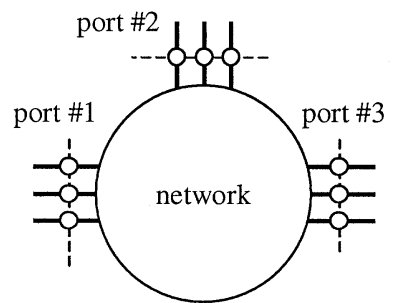

(b)
Fig. 1. Nonlinear time-periodic network, operating in its periodic steady state. (a) Single-port case. (b) Multi-port case.

part of the system outside the objective network must be included in the calculation, and the steady-state solution is assumed to be available at the outset from a harmonic power flow solution. A recent paper [6] reviews many of the best harmonic power flow solution methods presently available.

The state equations of the objective network, as seen from the port(s), can be derived in the following form [7]:

$$
\begin{aligned}
\frac{d x}{d t} & =f(x, u(t)) \\
y & =g(x, u(t))
\end{aligned}
$$

where $x \in \mathbf{R}^{N}, u, y \in \mathbf{R}^{N_{p h}}$ are state, input, output vectors, respectively, and $f \in \mathbf{R}^{N+N_{p h}} \rightarrow \mathbf{R}^{N}, g \in \mathbf{R}^{N+N_{p h}} \rightarrow$ $\mathbf{R}^{N_{p h}}$ nonlinear algebraic functions.

\section{B. Harmonic Domain State Equations}

Let us assume that a disturbance takes place outside the network causing excursions of $x, u, y$ from the steady state. The excursions are denoted as $\Delta x, \Delta u, \Delta y$, and assumed small enough to allow the linearization:

$$
\begin{aligned}
\frac{d(\Delta x)}{d t} & =\frac{\partial f}{\partial x} \Delta x+\frac{\partial f}{\partial u} \Delta u \equiv A_{t} \Delta x+B_{t} \Delta u \\
\Delta y & =\frac{\partial g}{\partial x} \Delta x+\frac{\partial g}{\partial u} \Delta u \equiv C_{t} \Delta x+D_{t} \Delta u .
\end{aligned}
$$

Because the coefficient matrices $A_{t}, B_{t}, C_{t}$, and $D_{t}$ are $T$-periodic due to the linearization, the above equations are the $T$-periodic linear state equations representing the network.

If we regard the network together with outside elements as one closed system, the transition matrix of the whole system is expressed as $\Phi(t, 0)=R(t) e^{Q t}$ based on Floquet theory [8]-[10], where $R(t)$ is a $T$-periodic and $Q$ a constant matrix. A particular mode of the transition matrix takes the simple form $e^{s t} r(t)$, where $s$ is the complex frequency and $r(t)$ a $T$-periodic vector [10]. Therefore, all variables $\Delta x, \Delta u$, and $\Delta y$ vary, for one mode, in unison according to the exponential modal pattern, and $\Delta x$ can be replaced by its $T$-periodic part $\Delta x_{t}$ multiplied by an exponential function of time: $\Delta x=\Delta x_{t} e^{s t}$. Expressing the $T$-periodic part by its Fourier series, we obtain

$$
\Delta x=\sum_{h=-\infty}^{\infty} \Delta x_{h} e^{\left(s+j h \omega_{0}\right) t} .
$$

The same applies to $\Delta u$ and $\Delta y$. The time derivative of (5) is given by

$$
\frac{d(\Delta x)}{d t}=e^{s t} \sum_{h=-\infty}^{\infty}\left(s+j h \omega_{0}\right) \Delta x_{h} e^{j h \omega_{0} t}
$$

The Fourier series of $A_{t}$ in (3) is

$$
A_{t}=\sum_{k=-\infty}^{\infty} A_{k} e^{j k \omega_{0} t}
$$

and the same applies to the other coefficient matrices $B_{t}, C_{t}$, and $D_{t}$ in (3) and (4). Substituting (5)-(7) into (3) and (4) gives

$$
\begin{aligned}
\sum_{h=-\infty}^{\infty}\left(s+j h \omega_{0}\right) \Delta x_{h} e^{j h \omega_{0} t}= & \left(\sum_{k=-\infty}^{\infty} A_{k} e^{j k \omega_{0} t}\right) \\
& \times\left(\sum_{h=-\infty}^{\infty} \Delta x_{h} e^{j h \omega_{0} t}\right) \\
& +\left(\sum_{k=-\infty}^{\infty} B_{k} e^{j k \omega_{0} t}\right) \\
& \times\left(\sum_{h=-\infty}^{\infty} \Delta u_{h} e^{j h \omega_{0} t}\right) \\
\sum_{h=-\infty}^{\infty} \Delta y_{h} e^{j h \omega_{0} t}= & \left(\sum_{k=-\infty}^{\infty} C_{k} e^{j k \omega_{0} t}\right) \\
& \times\left(\sum_{h=-\infty}^{\infty} \Delta x_{h} e^{j h \omega_{0} t}\right) \\
& +\left(\sum_{k=-\infty}^{\infty} D_{k} e^{j k \omega_{0} t}\right) \\
& \times\left(\sum_{h=-\infty}^{\infty} \Delta u_{h} e^{j h \omega_{0} t}\right) .
\end{aligned}
$$

Note that the common exponential term $e^{s t}$ has cancelled out. We now focus on the first summation regarding $A_{t}$ on the right-hand side of (8). It is expanded in ascending order with respect to the harmonics:

$$
\begin{aligned}
\cdots & +\sum_{h+k=-2} A_{k} \Delta x_{h} e^{-j 2 \omega_{0} t}+\sum_{h+k=-1} A_{k} \Delta x_{h} e^{-j \omega_{0} t} \\
& +\sum_{h+k=0} A_{k} \Delta x_{h}+\sum_{h+k=1} A_{k} \Delta x_{h} e^{j \omega_{0} t} \\
& +\sum_{h+k=2} A_{k} \Delta x_{h} e^{j 2 \omega_{0} t}+\cdots
\end{aligned}
$$

By neglecting the $N_{h}$-th and higher harmonics of $\Delta x_{t}$ and also neglecting $N_{k}$-th and higher harmonic components of $A_{t}$, the coefficients of the exponential terms can be expressed by the following matrix notation (for $N_{k}=3$ ):

$$
\left[\begin{array}{ccccccc}
A_{0} & A_{-1} & A_{-2} & & & & \\
A_{1} & A_{0} & A_{-1} & \cdot & & 0 & \\
A_{2} & A_{1} & A_{0} & & \cdot & & \\
& \cdot & & \cdot & & \cdot & \\
& & \cdot & & A_{0} & A_{-1} & A_{-2} \\
& 0 & & \cdot & A_{1} & A_{0} & A_{-1} \\
& & & & A_{2} & A_{1} & A_{0}
\end{array}\right]\left[\begin{array}{c}
\Delta x_{-}\left(N_{h}-1\right) \\
\vdots \\
\Delta x_{-1} \\
\Delta x_{0} \\
\Delta x_{1} \\
\vdots \\
\Delta x_{N_{h}-1}
\end{array}\right]
$$$$
\equiv A \Delta x \text {. }
$$ 
If $A_{t}$ is a scalar, then the above defined $A$ is a Hermitian Toeplitz matrix. If $A_{t}$ is a matrix, then $A$ is a "block" Hermitian Toeplitz matrix, consisting of the Fourier coefficients of $A_{t}$. Defining $M$ as

$$
M \equiv 2 N_{h}-1
$$

the size of $A$ is $M$ by $M$, and its nonzero bandwidth is $2 N_{k}-1$. The harmonic domain state vector, consisting of the Fourier coefficients of $\Delta x_{t}$, is denoted by $\Delta x \in \mathbf{C}^{N M}$ [to be distinguished from its time domain counterpart in (3) and (4)]. Applying this matrix notation to all terms on the right-hand sides of (8) and (9), we obtain the harmonic domain state equations

$$
\begin{aligned}
S \Delta x & =A \Delta x+B \Delta u \\
\Delta y & =C \Delta x+D \Delta u
\end{aligned}
$$

where $\Delta u, \Delta y \in \mathbf{C}^{N_{p h} M}$ are the harmonic domain input and output vectors, and $S$ is the matrix of dynamic differentiation with respect to time, in the harmonic domain, defined by

$$
S=\operatorname{diag}\left(\left\{s+j h \omega_{0}\right\} I\right)
$$

with $h=-\left(N_{h}-1\right), \ldots,-1,0,1, \ldots, N_{h}-1$ and $I$ is the $N$ by $N$ identity matrix. The above definition of $S$ includes not only the harmonic frequency $h \omega_{0}$ but also the complex frequency $s$ to express the dynamics of the network, and thus, this is an extension of the static differentiation matrix

$$
S_{\text {static }}=\operatorname{diag}\left(j h \omega_{0} I\right)
$$

proposed in [3] and [4].

\section{Harmonic Domain Dynamic Transfer Function}

Substituting (13) into (14) gives the harmonic input-output relation

$$
\Delta y=\left\{C(S-A)^{-1} B+D\right\} \Delta u .
$$

Thus, the HDDTF of the network is

$$
H(s) \equiv C(S-A)^{-1} B+D
$$

where $H(s)$ is an $M$ by $M$ complex matrix. Because $S$ is a differentiation operator applied to each harmonic component of the state vector, the HDDTF is a transfer-function matrix relating the harmonic domain input and output vectors $\Delta u$ and $\Delta y$ endowed with $s$-domain properties, in other words, it characterizes the dynamics of the network in terms of the frequency response of the harmonic perturbations superimposed on the periodic steady state. The $(p, q)$ element $h_{p q}(s)$ of $H(s)$ relates the $q$-th harmonic input to the $p$-th harmonic output, and therefore, $h_{p q}(s)$ is the frequency response of the harmonic coupling between those input and output (note that $h_{00}(s)$ is located at the center of $H(s)$ ). Practically, we can calculate the HDDTF matrix at a frequency $f$ by substituting $s=j \omega=j 2 \pi f$ into (15) and then $S$ into (18). Note that $h \omega_{0}$ stands for harmonics and $\omega$ for (angular) frequency.

The above derivation of HDDTF is applicable to the multiphase case, where $N_{p h}$ is bigger than one. The most common three-phase case is used here for illustration. The input vector takes the form $\Delta u=\left[\begin{array}{lll}\Delta u_{a} & \Delta u_{b} & \Delta u_{c}\end{array}\right]^{T}$ in the time domain, and in the harmonic domain the harmonics of $\Delta u_{a}, \Delta u_{b}, \Delta u_{c}$ are arranged as

$$
\begin{aligned}
& \Delta u=\left[\Delta u_{a,-\left(N_{h}-1\right)} \quad \Delta u_{b,-\left(N_{h}-1\right)} \quad \Delta u_{c,-\left(N_{h}-1\right)}\right. \\
& \text {... } \\
& \Delta u_{a,-1} \quad \Delta u_{b,-1} \quad \Delta u_{c,-1} \\
& \Delta u_{a, 0} \quad \Delta u_{b, 0} \quad \Delta u_{c, 0} \\
& \Delta u_{a, 1} \quad \Delta u_{b, 1} \quad \Delta u_{c, 1} \\
& \text {... } \\
& \left.\Delta u_{a, N_{h}-1} \quad \Delta u_{b, N_{h}-1} \quad \Delta u_{c, N_{h}-1}\right]^{T} .
\end{aligned}
$$

The same applies to the output vector $\Delta y$.

If the port voltages and currents are chosen as $u$ and $y$ respectively, such an HDDTF is a harmonic domain dynamic admittance matrix (HDDAM), which is compatible with admittanceand conductance-based methods widely used in power system analysis.

\section{Modal ANALYSIS OF HDDTF}

Once the $M$ by $M$ HDDTF matrix $H(s)$ has been calculated, the results should be interpreted in a meaningful way so as to facilitate any useful application of the obtained information. A first and simple description of the results is of course directly available by representing the individual elements of $H(s)$ defined in (18). However, $M^{2}$, the number of such elements is large and their significance is somewhat obscured by the operations of both multiplication and addition in the relation of input harmonics to output harmonics. Nevertheless, some resonance effects may appear even in this type of representation as shown in Section IV.

\section{A. Modal Decomposition of HDDTF}

It is more natural to characterize the HDDTF by a modal decomposition (i.e., by using its eigenvalues and eigenvectors). We start with the harmonic input-output relation (17) combined with (18)

$$
\Delta y=H(s) \Delta u .
$$

Assuming that $H(s)$ is diagonalizable, (20) gives

$$
\Delta y=P \operatorname{diag}\left(\lambda_{1}, \lambda_{2}, \ldots, \lambda_{M}\right) P^{-1} \Delta u
$$

where $\lambda_{1}, \lambda_{2}, \ldots, \lambda_{M}$ are the eigenvalues of $H(s)$, and the transformation matrix $P$ is given by the eigenvectors $v_{1}, v_{2}, \cdots$, $v_{M}: P=\left[\begin{array}{llll}v_{1} & v_{2} & \cdots & v_{M}\end{array}\right]$. Note that the eigenvalues and the eigenvectors (and thus, the transformation matrix) are all functions of $s$. The modal input is defined by $\Delta \hat{u}=P^{-1} \Delta u$, and the modal output by $\Delta \hat{y}=P^{-1} \Delta y$. Thus, (21) yields $\Delta \hat{y}=\operatorname{diag}\left(\lambda_{1}, \lambda_{2}, \ldots, \lambda_{M}\right) \Delta \hat{u}$, or, element by element:

$$
\Delta \hat{y}_{m}=\lambda_{m}(s) \Delta \hat{u}_{m} \quad(m=1,2, \ldots, M) .
$$

In the above equation, couplings among different orders of harmonics are eliminated, and thus, the $M$ by $M$ matrix problem (20) is now decoupled into $M$ independent scalar relations (22). 


\section{B. Modal Characterization of HDDTF}

When the input $\Delta u$ is an eigenvector $v_{m}$ of $H(s)$ in (20), we obtain

$$
\Delta y_{m}(s)=\lambda_{m}(s) v_{m}(s)
$$

which shows that the vectors $v_{m}$ and $\Delta y_{m}$ are simply proportional to each other due to the scalar multiplier $\lambda_{m}(s)$. This relation is of particular significance in the case of an HDDTF, because the inputs and outputs in (20) and (23) are vectors of harmonic components, and thus, they represent $T$-periodic waveshapes in the time domain. In particular, an eigenvector $v_{m}$ (normalized to unit length) represents a modal $T$-periodic waveshape or characteristic $T$-periodic waveshape (of fully defined magnitude). Thus, if the input includes the characteristic waveshape given by $v_{m}$, that component in the output will have the same shape but amplified by the corresponding eigenvalue $\lambda_{m}(s)$. We note that the characteristic waveshape is generally a complex-valued quantity in the time domain, and superposition of all modal components gives real-valued inputs and outputs as expressed in $\Delta u=P \Delta \hat{u}$ and $\Delta y=P \Delta \hat{y}$. The variation of the elements of an eigenvector with respect to frequency, in other words, the variation of the harmonic components in a characteristic waveshape with respect to frequency, is shown in Section III-C. More interesting is however the variation of the corresponding eigenvalue $\lambda_{m}(s)$ with respect to frequency, since $\lambda_{m}(s)$ may have sharp peaks in its frequency variation. This suggests a resonance phenomenon and potential loss of stability, since a nearby pole responsible for strong resonance may be sensitive to the system parameters and could possibly cross the imaginary axis.

\section{Half-Wave Symmetry}

In ac power networks, nonlinear and time-periodic components often result in only even harmonics in the elements of the $A_{t}, B_{t}, C_{t}, D_{t}$ matrices of (3) and (4). In such a case, if the driving force is purely sinusoidal, the steady-state waveforms of the variables $x, u$, and $y$ consist of only odd harmonics, in other words, the network exhibits "half-wave symmetry."

When the network exhibits half-wave symmetry, the $A, B$, $C, D$ matrices in (13) and (14) take the following form:

$\left[\begin{array}{cccccccccc}A_{0} & 0 & A_{-2} & 0 & A_{-4} & & & & \\ 0 & A_{0} & 0 & A_{-2} & 0 & A_{-4} & & & 0 \\ A_{2} & 0 & A_{0} & 0 & A_{-2} & 0 & A_{-4} & & \\ 0 & A_{2} & 0 & A_{0} & 0 & A_{-2} & 0 & A_{-4} & \\ A_{4} & 0 & A_{2} & 0 & A_{0} & 0 & A_{-2} & 0 & . \\ & A_{4} & 0 & A_{2} & 0 & A_{0} & 0 & . & & . \\ & & A_{4} & 0 & A_{2} & 0 & . & & . \\ & & & A_{4} & 0 & . & & . & \\ & 0 & & & . & & . & & \end{array}\right]$

(for $N_{k}=5$ ), because no odd harmonic components are present. From (13) and (14), and (24), it can be confirmed that the odd and the even harmonics in $\Delta x, \Delta u$, and $\Delta y$ are decoupled from each other, and thus, harmonic inputs of odd orders produce

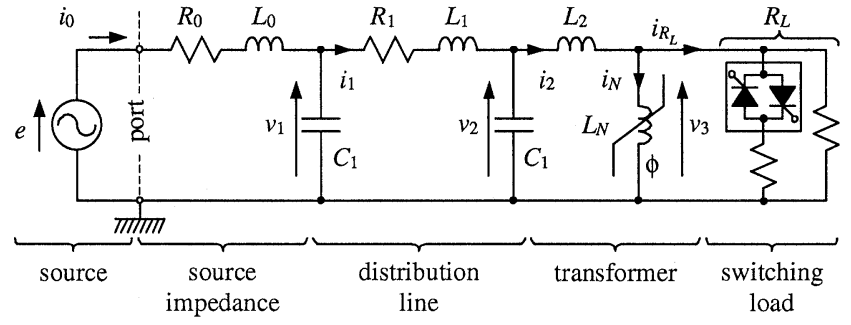

Fig. 2. Test Network 1. Network parameters are $e=E_{m} \cos \left(\omega_{0} t\right), \omega_{0}=$ $2 \pi 60[\mathrm{rad} / \mathrm{s}], E_{m}=\sqrt{ }(2 / 3) \times V_{p u} \times 15[\mathrm{kv}], R_{0}=1[\Omega], L_{0}=20[\mathrm{mH}]$, $R_{1}=12.7[\Omega], L_{1}=94.9[\mathrm{mH}], C_{1}=0.201[\mu \mathrm{F}], L_{2}=20[\mathrm{mH}]$, $L_{N}: i_{N}=\alpha \phi+\beta \phi^{7}, \alpha=0.01, \beta=5 \times 10^{-10}$, and $R_{L}=100 / 200[\Omega]$ (on/off).

outputs of odd orders only and vice versa. Therefore, by rearranging the elements of $\Delta u$ and $\Delta y$ (first all odd harmonics and then all even harmonics), the HDDTF of (18) becomes

$$
H=\left[\begin{array}{cc}
H_{\text {odd }} & 0 \\
0 & H_{\text {even }}
\end{array}\right] .
$$

Thus, the eigenvalues of $H$ consist of those of $H_{\text {odd }}$ and $H_{\text {even }}$. In this paper, the eigenvalues (and the associated eigenvectors) of $H_{\text {odd }}$ are called "odd modes" and those of $H_{\text {even }}$ "even modes." An eigenvalue $\lambda_{m}$ of $H$ satisfies the characteristic equation of $H$ with its eigenvector $v_{m}$

$$
\left(H-\lambda_{m} I\right) v_{m}=0 .
$$

Substituting (25) into (26) gives

$$
\left[\begin{array}{cc}
H_{\text {odd }}-\lambda_{m} I & 0 \\
0 & H_{\text {even }}-\lambda_{m} I
\end{array}\right]\left[\begin{array}{c}
v_{\text {odd }} \\
v_{\text {even }}
\end{array}\right]=0 .
$$

If the eigenvalue $\lambda_{m}$ is of an odd mode, it also satisfies the characteristic equation of $H_{\text {odd }},\left(H_{\text {odd }}-\lambda_{m} I\right) v_{\text {odd }}=0$, but does not satisfy that of $H_{\text {even }}$. This requires $v_{\text {even }}=0$ in (27). If $\lambda_{m}$ is of an even mode, then $v_{\text {odd }}=0$. Thus, the transformation matrix $P$ also exhibits decoupling between the odd and even harmonics as in (25).

The above results confirm the following features of $H(s)$ in the case of half-wave symmetry: (i) The odd and the even harmonics in input and output are decoupled from each other; (ii) The eigenvalues of $H(s)$ consist of two different groups of eigenvalues, odd modes and even modes, where the former relate odd inputs to odd outputs and the latter relate even inputs to even outputs; (iii) The transformation matrix $P$ has the sparse structure of the decoupling, and the characteristic waveshapes defined by the eigenvectors of the odd modes represent odd waveforms and those of even modes represent even waveforms.

\section{NUMERICAL EXAMPLES}

\section{A. Test Network 1}

Fig. 2 shows a small Test Network including both nonlinear and time-periodic components. The network contains, from left to right, a source, source impedance, a distribution line, a transformer, and a switching load. The voltage source $e$ represents a $60-\mathrm{Hz}, 15-\mathrm{kV}$ sinusoidal bus voltage whose per-unit amplitude is given by $V_{p . u}$. as in the figure caption. In this analysis, we 

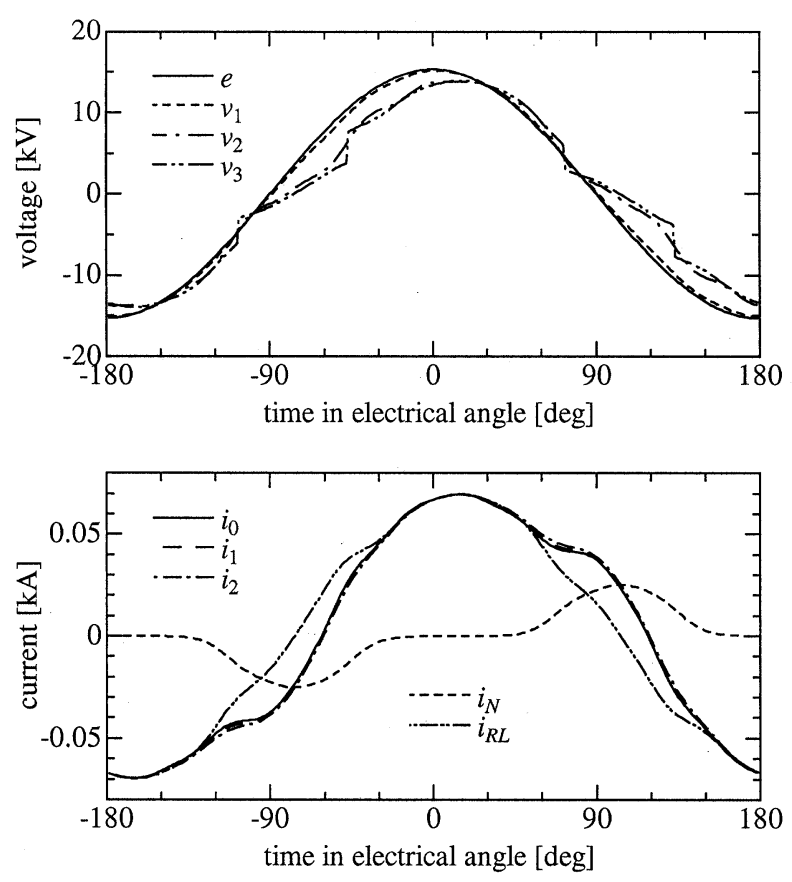

Fig. 3. Steady-state solution of Test Network 1.

assume a temporary overvoltage of $V_{p . u .}=1.25$. The source impedance is represented by $R_{0}$ and $L_{0}$. The distribution line is represented by its $\pi$ equivalent consisting of $R_{1}, L_{1}, C_{1}$, and the transformer by its leakage inductance $L_{2}$ and magnetizing inductance $L_{N} . L_{N}$ is nonlinear and represents the saturation characteristic of the magnetizing branch by a 7-th order expression $i_{N}=\alpha \phi+\beta \phi^{7}$, where $\phi$ and $i_{N}$ denote the magnetic flux and the current. The switching load $R_{L}$ consists of two linear time-invariant resistors, both $200 \Omega$, and a switch comprised of forced commuted devices. Here we define $\theta$ as the phase angle of the fundamental harmonic of $v_{3}$. The switch is on, and thus, $R_{L}=100 \Omega$, when $-2 \pi / 3<\theta<-\pi / 3$ and $\pi / 3<\theta<2 \pi / 3$. Otherwise, it is off and $R_{L}=200 \Omega$. Therefore, $R_{L}$ is a $T$-periodic resistance alternating between 100 and $200 \Omega$ due to the $T$-periodic switching.

The network is seen from the left-hand side of the port, and the voltage applied to the network $e$ and the current injected into the network $i_{0}$ are regarded as the input $u$ and the output $y$, respectively. By selecting the state variables $x_{1}=i_{0}, x_{2}=v_{1}$, $x_{3}=i_{1}, x_{4}=v_{2}, x_{5}=i_{2}, x_{6}=\phi$, we obtain the state and the output equations of the network corresponding to (1) and (2):

$$
\begin{aligned}
\frac{d}{d t}\left[\begin{array}{c}
x_{1} \\
x_{2} \\
x_{3} \\
x_{4} \\
x_{5} \\
x_{6}
\end{array}\right] & =\left[\begin{array}{c}
-\frac{R_{0}}{L_{0}} x_{1}-\frac{1}{L_{0}} x_{2}+\frac{1}{L_{0}} u \\
\frac{1}{C_{1}} x_{1}-\frac{1}{C_{1}} x_{3} \\
\frac{1}{L_{1}} x_{2}-\frac{R_{1}}{L_{1}} x_{3}-\frac{1}{L_{1}} x_{4} \\
\frac{1}{C_{1}} x_{3}-\frac{1}{C_{1}} x_{5} \\
\frac{1}{L_{2}} x_{4}-\frac{R_{L}}{L_{2}} x_{5}+\frac{R_{L}}{L_{2}}\left(\alpha x_{6}+\beta x_{6}^{7}\right) \\
R_{L} x_{5}-R_{L}\left(\alpha x_{6}+\beta x_{6}^{7}\right)
\end{array}\right] \\
y & =x_{1} .
\end{aligned}
$$

Fig. 3 shows the steady-state solution, obtained by (28) and (29) using the method proposed in [3], considering up to 11-th harmonic both in the state variables and in the system coefficients

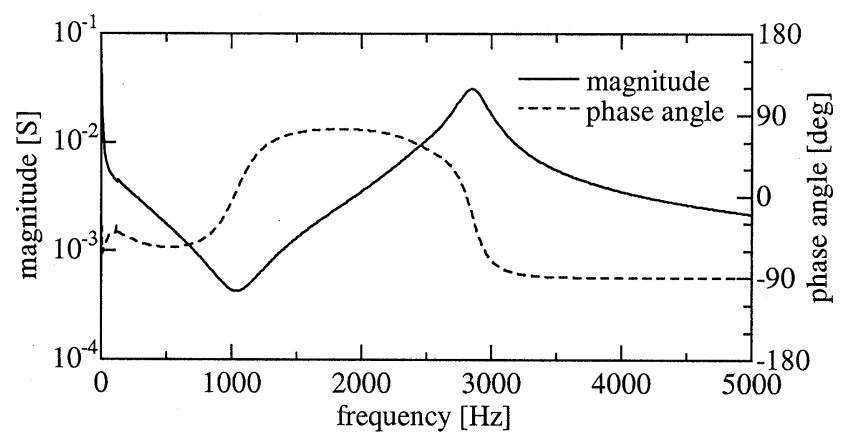

(a) $(0,0)$ element

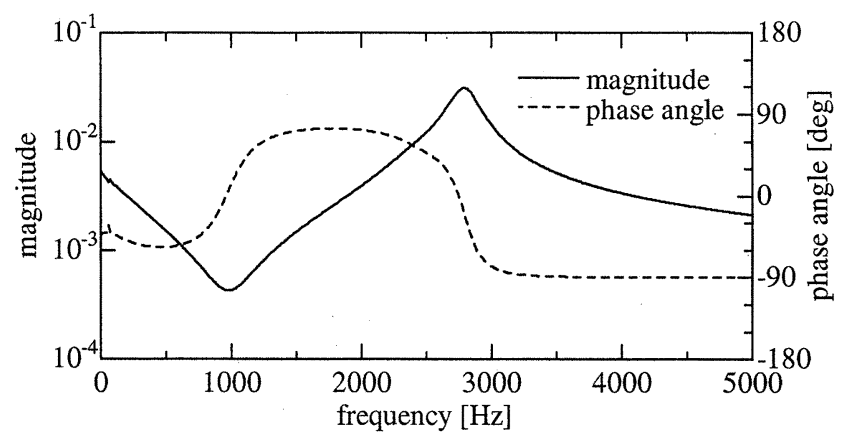

(b) $(1,1)$ element

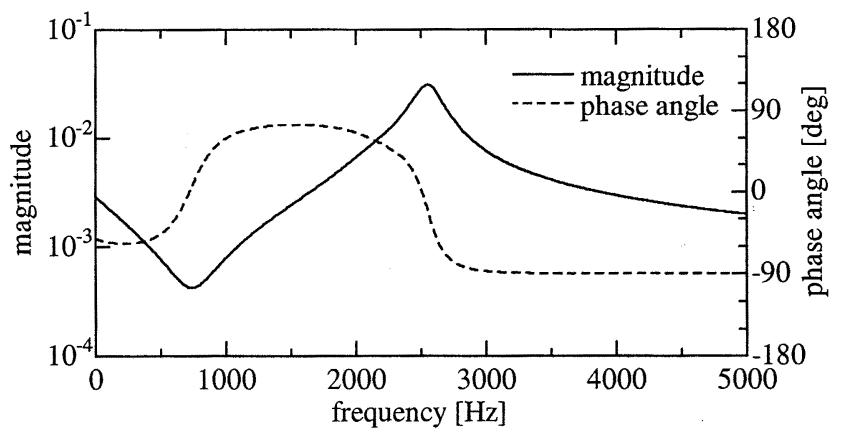

(c) $(5,5)$ element

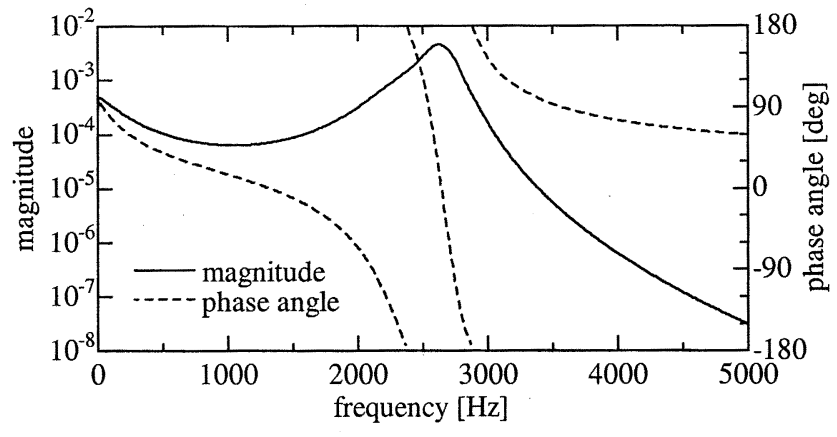

(d) $(2,4)$ element

Fig. 4. Frequency responses of HDDTF elements for Test Network 1. Elements (a) $(0,0),(b)(1,1),(c)(5,5)$, and $(d)(2,4)$ are shown, where element $(p, q)$ denotes $h_{p q}(s)$ of $H(s)$ that relates $q$-th harmonic input to $p$-th harmonic output.

$\left(N_{h}=N_{k}=12, M=23\right)$. Both the nonlinear inductor $L_{N}$ and the $T$-periodic resistor $R_{L}$ create harmonics in the waveforms. It can be confirmed that the waveforms are almost sinusoidal when $V_{p u}=1$ and the switch is always on or off, although the result is not shown here. 


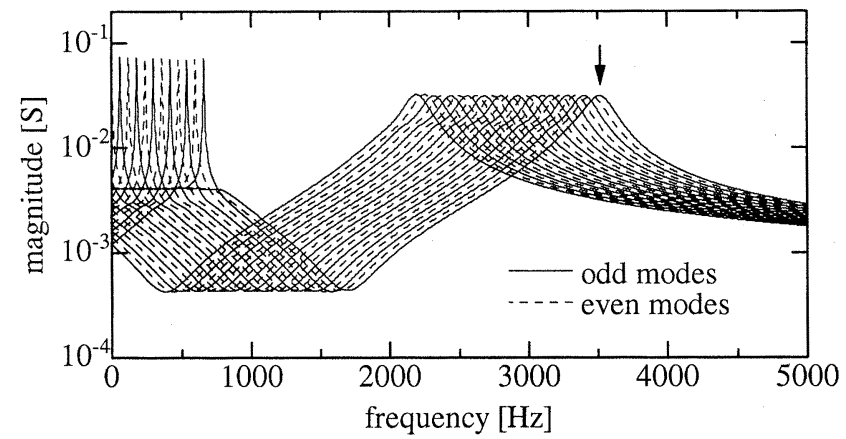

(a)

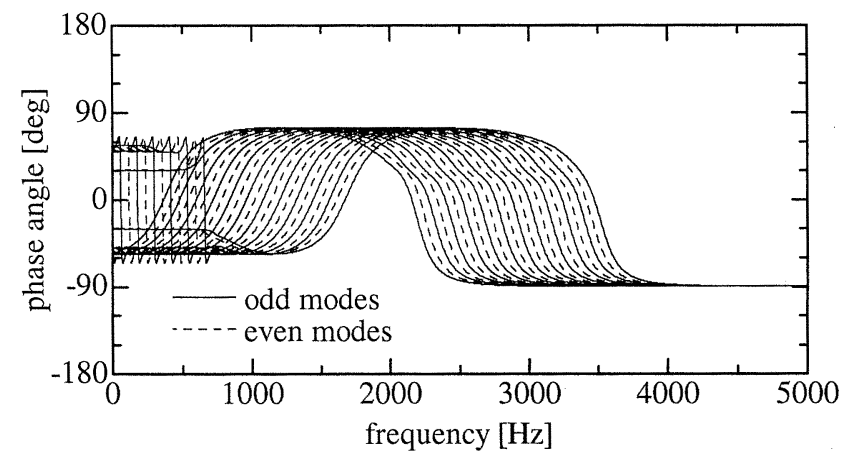

(b)

Fig. 5. Variation of eigenvalues with respect to frequency for Test Network 1. (a) Magnitude. (b) Phase angle.

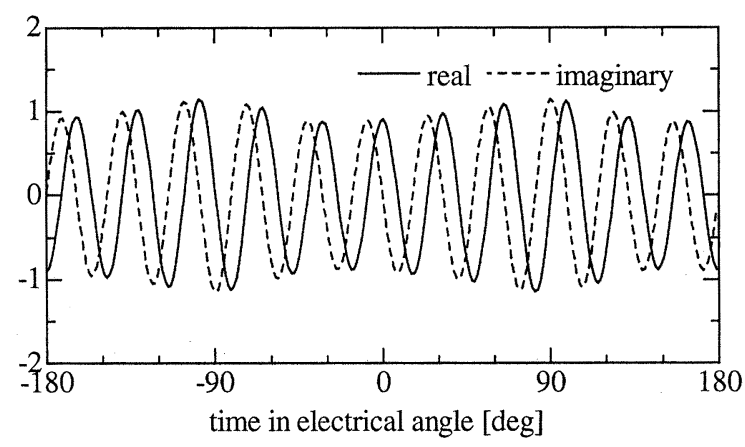

Fig. 6. Example of characteristic waveshape for Test Network 1. This eigenvector is taken from the first column of $P$ at $f=3.509 \mathrm{kHz}$.

The linearization of (3) and (4) applied to (28) and (29), about the $T$-periodic steady-state solution, provides the following $T$-periodic coefficient matrices:

$A_{t}=\left[\begin{array}{cccccc}-\frac{R_{0}}{L_{0}} & -\frac{1}{L_{0}} & 0 & 0 & 0 & 0 \\ \frac{1}{C_{1}} & 0 & -\frac{1}{C_{1}} & 0 & 0 & 0 \\ 0 & \frac{1}{L_{1}} & -\frac{R_{1}}{L_{1}} & -\frac{1}{L_{1}} & 0 & 0 \\ 0 & 0 & \frac{1}{C_{1}} & 0 & -\frac{1}{C_{1}} & 0 \\ 0 & 0 & 0 & \frac{1}{L_{2}} & -\frac{R_{L}}{L_{2}} & \frac{R_{L}}{L_{2}}\left(\alpha+7 \beta x_{6}^{6}\right) \\ 0 & 0 & 0 & 0 & R_{L} & -R_{L}\left(\alpha+7 \beta x_{6}^{6}\right)\end{array}\right]$ $B_{t}=\left[\begin{array}{llllll}\frac{1}{L_{0}} & 0 & 0 & 0 & 0 & 0\end{array}\right]^{T}$

$C_{t}=\left[\begin{array}{llllll}1 & 0 & 0 & 0 & 0 & 0\end{array}\right] \quad D_{t}=0$.

The elements $(5,5),(5,6),(6,5)$, and $(6,6)$ of $A_{t}$ are $T$-periodic, because they include $x_{6}$ and $R_{L}$, being $T$-periodic in the steady state. The Hermitian Toeplitz matrices $A, B, C$, and $D$

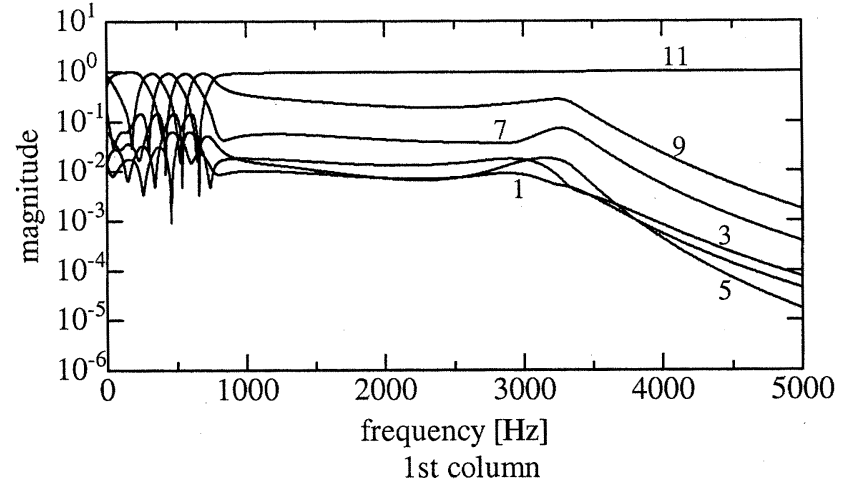

(a)

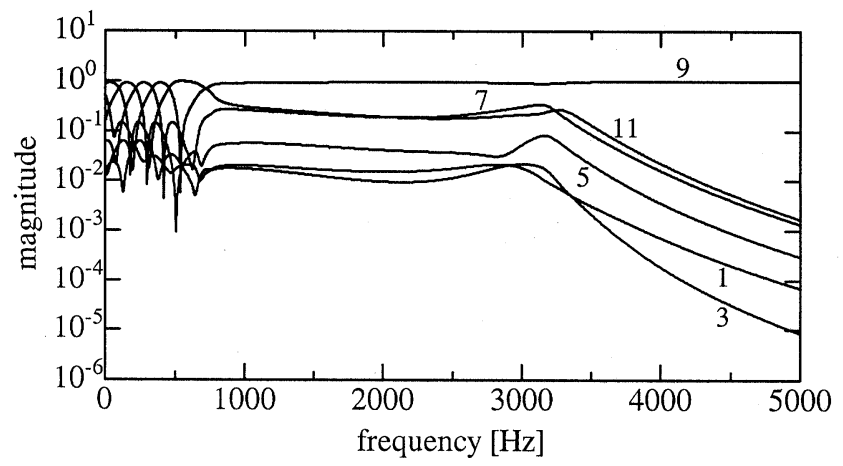

(b)

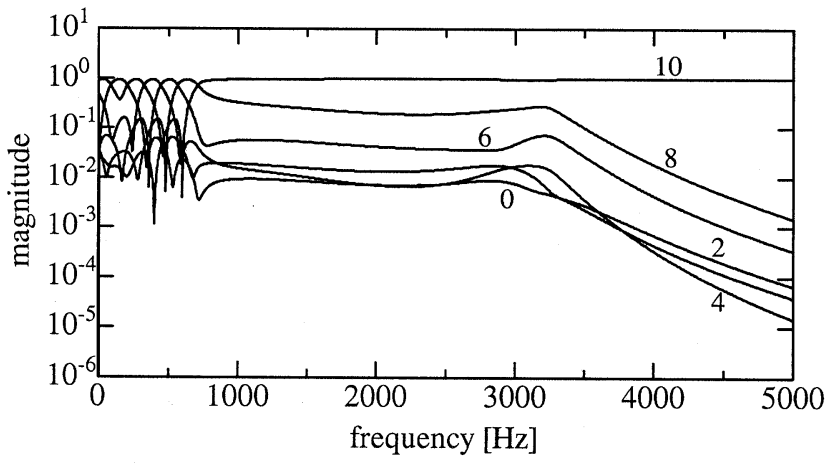

(c)

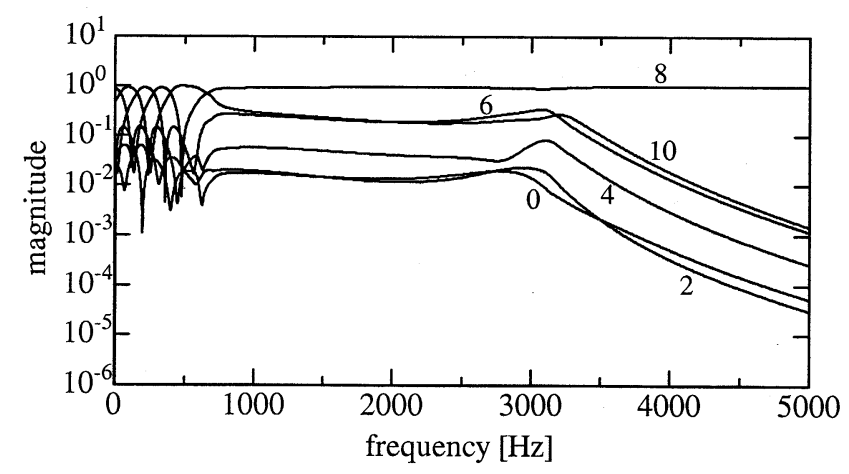

(d)

Fig. 7. Variation of elements of eigenvectors (harmonic components of characteristic waveshapes) with respect to frequency for Test Network 1. Subplots (a), (b), (c), and (d) are the first, second, 13-th, and 14-th columns of $P$. The harmonic orders are marked by numbers.

of (13) and (14) are built as in (11) from the above $A_{t}, B_{t}, C_{t}$, and $D_{t}$, and substituted into (18) to obtain the HDDTF matrix 


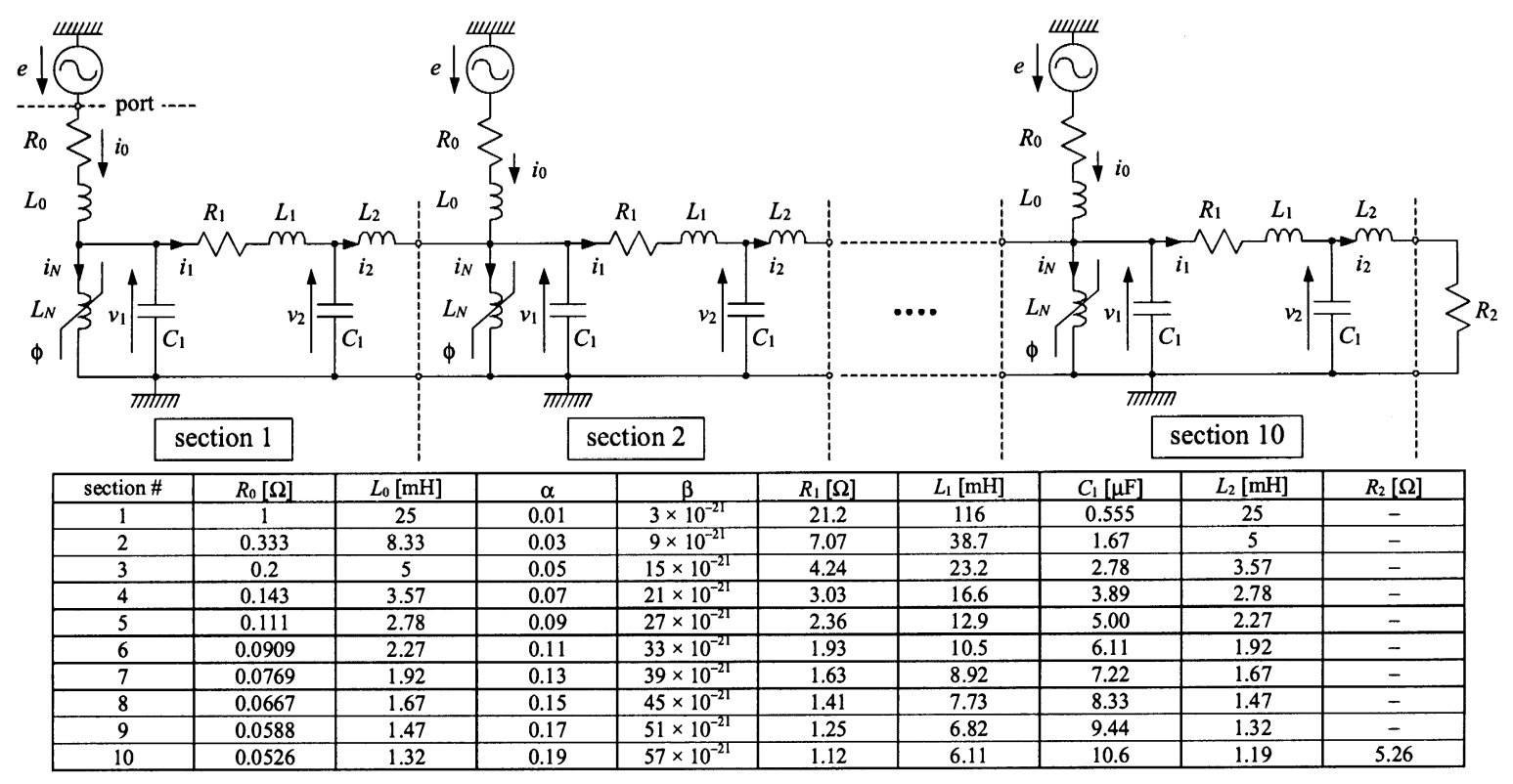

Fig. 8. Test Network 2, consisting of ten sections. Network parameters are shown in the above table except $e=E_{m} \cos \left(\omega_{0} t\right), \omega_{0}=2 \pi 60[\mathrm{rad} / \mathrm{s}]$, and $E_{m}=\sqrt{ }(2 / 3) \times V_{p . u} . \times 500[\mathrm{kV}]$.

$H(s)$ of Test Network 1. Fig. 4 shows the frequency responses of elements in $H(s)$. Because the number of elements in $H(s)$ is large $\left(M^{2}=529\right)$, only four elements are shown. From the comparison of the diagonal elements shown in Fig. 4(a)-(c), a uniform shift of multiples of $\omega_{0}$ is observed; the shift between (a) and (b) is $-\omega_{0}$ and between (a) and (c) is $-5 \omega_{0}$. This corresponds to the known concept of "signal modulation" conveyed by (15), where the frequency of the harmonics $h \omega_{0}$ is modulated by the frequency used to represent the dynamics of the network $\omega$. Fig. 4(d) shows the off-diagonal element $h_{24}(s)$. Note that $h_{p q}(s)=0$ if $q=p \pm 1$ due to the decoupling (25) of the odd and even harmonics. Fig. 5 shows the variation of the eigenvalues of $H(s)$ with respect to frequency. The odd and even modes alternate with each other by $\omega_{0}$, and two independent groups of resonance are observed. Fig. 6 shows the characteristic waveshape of an odd mode at $f=3.509 \mathrm{kHz}$, a resonance peak marked by the arrow in Fig. 5(a). Fig. 7 shows the variation of the elements of eigenvectors (i.e., the variation of the harmonic components of characteristic waveshapes with respect to frequency), where the positive $p$-th and the negative $p$-th harmonic components are combined into their 2-norms. Two odd modes (a), (b) (the first and the second columns of the transformation matrix $P$ ) and two even modes (c), (d) (the 13-th and the 14-th columns) are shown. Since the characteristic waveshape of Fig. 6 is taken from the first column of $P$, Fig. 7(a) corresponds to Fig. 6. At the resonance frequency $f=3.509 \mathrm{kHz}$ in Fig. 7(a), the 11-th harmonic is dominant $(=1)$, and therefore, the characteristic waveshape has a dominant 11-th harmonic as in Fig. 6. Each set of eigenvalue and eigenvector is tracked in the frequency variations of Figs. 5 and 7 to avoid eigenvalue crossovers.

\section{B. Test Network 2}

Fig. 8 shows a relatively large Test Network consisting of ten sections. Each section has a voltage source $e$, linear time-invariant elements $R_{0}, L_{0}, R_{1}, L_{1}, C_{1}, L_{2}$, and a nonlinear inductor $L_{N}$. The number of state variables for each section is 6 , and thus, the total number of state variables for the whole network is 60 . The voltage source $e$ represents a $60-\mathrm{Hz}, 500-\mathrm{kV}$ sinusoidal bus voltage whose per-unit amplitude is given by $V_{p . u}$. in the same way as in Test Network 1. Here also we assume $V_{p . u .}=1.25$. The nonlinear inductor $L_{N}$ represents the magnetizing branch of a transformer, with its iron-core saturation characteristic represented by the same expression $i_{N}=\alpha \phi+\beta \phi^{7}$ as in Test Network 1. The $\pi$ equivalent consisting of $R_{1}, L_{1}$, and $C_{1}$ represents a 500-kV transmission line. Every section has different parameter values, shown in the table and the caption of Fig. 8.

The network is observed from the voltage source $e$ of the first section, and its voltage $e$ and current $i_{0}$ are considered as the input $u$ and the output $y$. Fig. 9 shows the steady-state solution obtained using the same method as in Test Network $1\left(N_{h}=N_{k}=12, M=23\right)$. The nonlinear inductors are saturated by the overvoltage. The state equations of the network are linearized, based on (3) and (4), about the $T$-periodic steady-state solution, and the HDDTF $H(s)$ is calculated by (18). Fig. 10 shows diagonal and offdiagonal elements $h_{00}(s)$ and $h_{13}(s)$ of $H(s)$, respectively. If the diagonal element $h_{00}(s)$ is compared with other diagonal elements, a uniform shift of multiples of $\omega_{0}$ is observed. The same applies to the off-diagonal elements. Because the number of state variables of Test Network 2 is 10 times larger than that of Test Network 1, Fig. 10 demonstrates a larger number of resonance peaks than that of Fig. 4, particularly within 1 to $1.5 \mathrm{kHz}$. Fig. 11 shows the variation of the eigenvalues of $H(s)$ as a function of frequency. Fig. 12 shows the variation of the elements of the first and the 13-th eigenvectors (the first and the 13-th columns of $P$ ) as function of frequency. Fig. 12 reveals that the first eigenvector has a dominant 11-th harmonic and the 13-th eigenvector a dominant 10-th harmonic. These dominant harmonics are also observed in the other eigenvectors but are not shown here. Thus, in this test case, each mode carries mainly the information of one particular harmonic, and the transformation matrix $P$ closely resembles a 

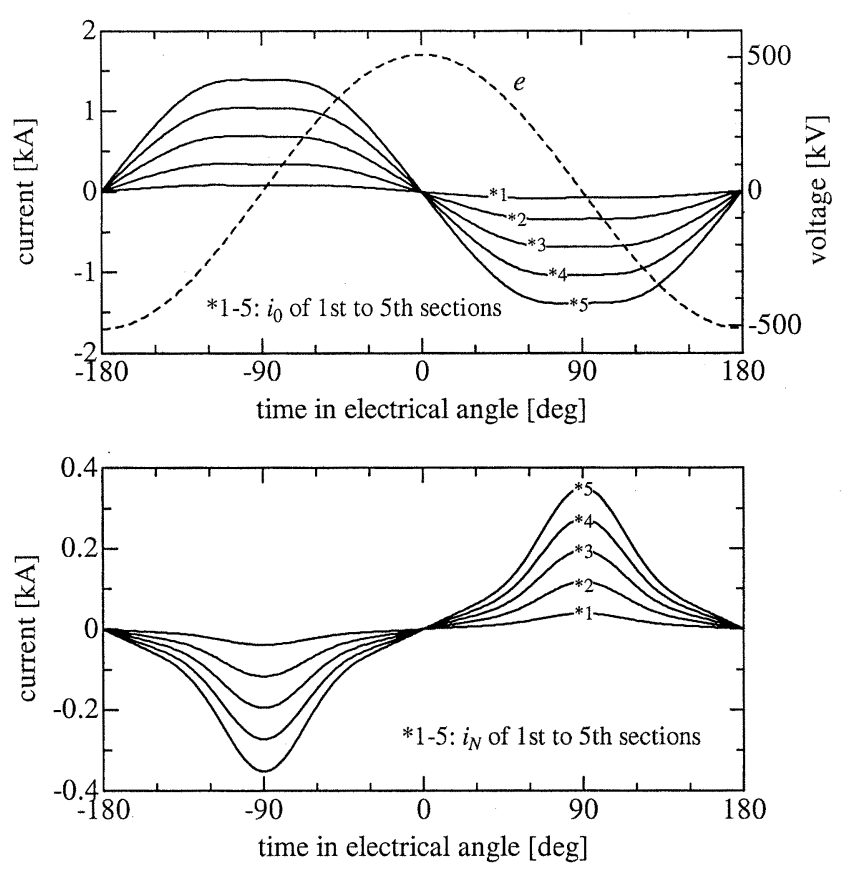

Fig. 9. Steady-state solution of Test Network 2.

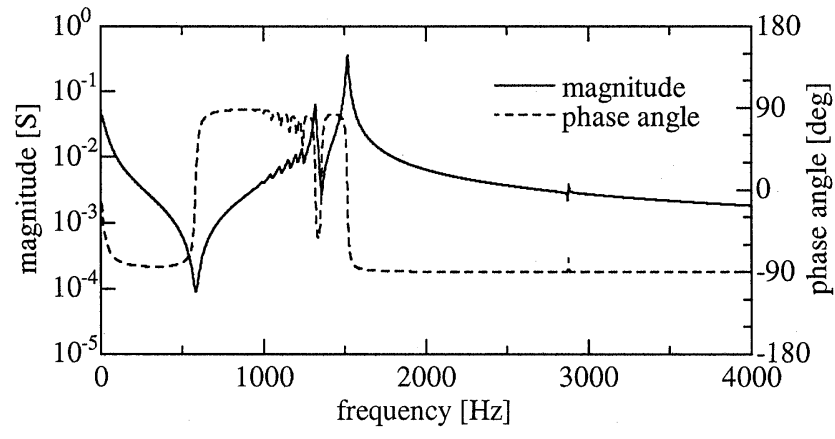

(a)

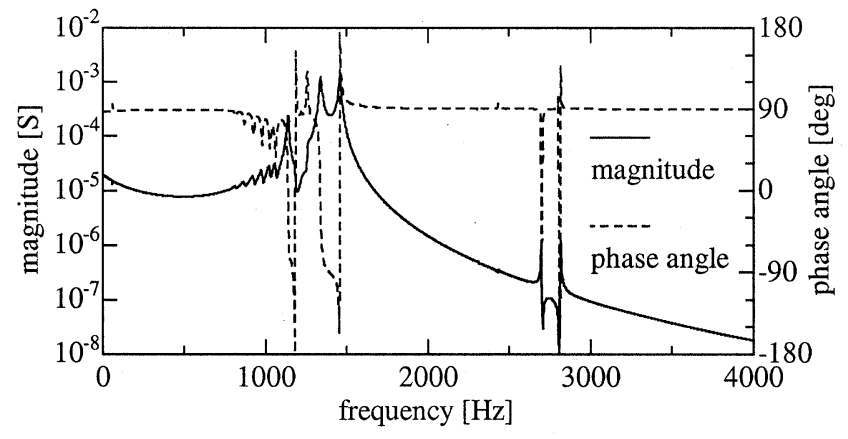

(b)

Fig. 10. Frequency responses of HDDTF elements for Test Network 2. Elements (a) $(0,0)$ and (b) $(1,3)$ are shown, where element $(p, q)$ denotes $h_{p q}(s)$ of $H(s)$ that relates $q$-th harmonic input to $p$-th harmonic output.

permutation matrix. The frequency variation of the eigenvalue that carries mainly the information of the 0 -th harmonic is taken from Fig. 11 and shown independently in Fig. 13(a), where resonance and antiresonance points are marked by "A" and "B," respectively. Fig. 13(b) shows the corresponding resonance and

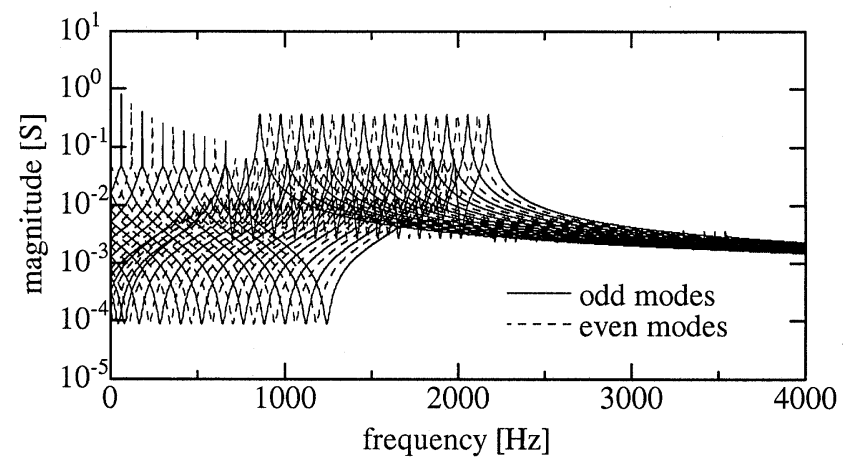

(a)

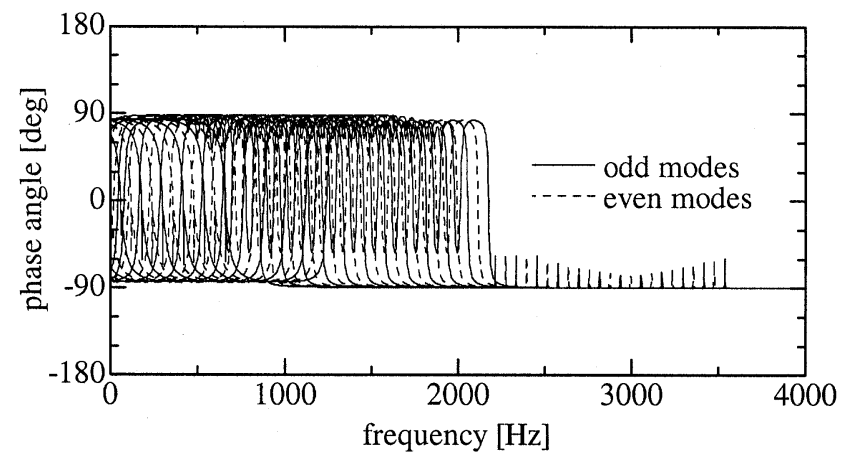

(b)

Fig. 11. Variation of eigenvalues with respect to frequency for Test Network 2. (a) Magnitude. (b) Phase angle.

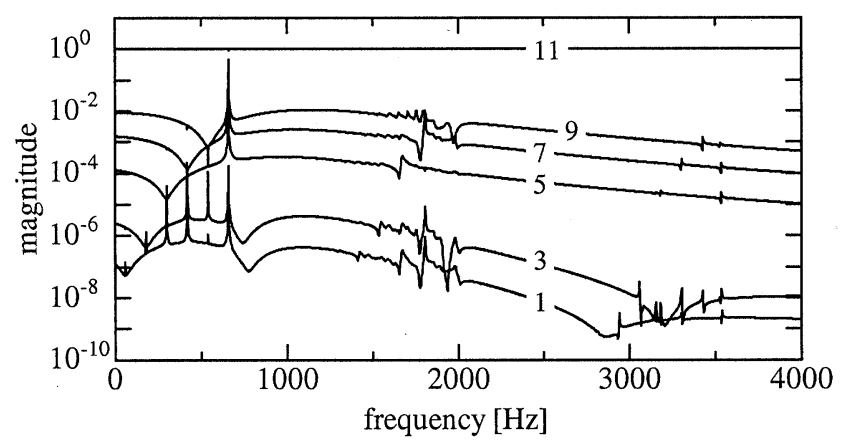

(a)

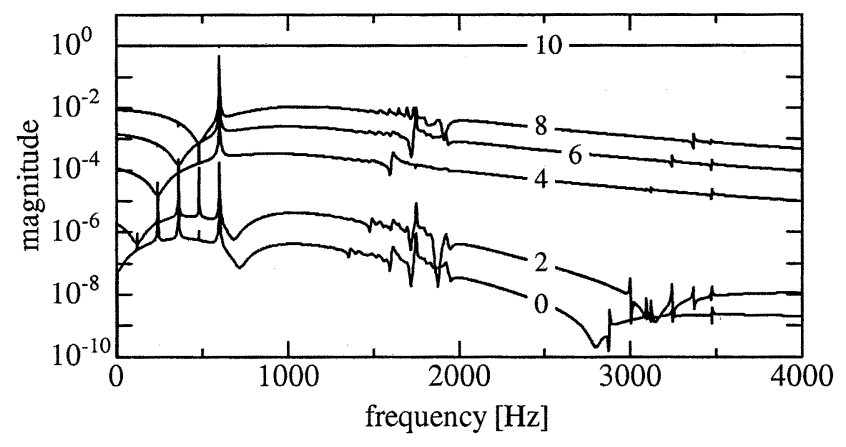

(b)

Fig. 12. Variation of elements of eigenvectors (harmonic components of characteristic waveshapes) with respect to frequency for Test Network 2. Subplots (a) and (b) are the first and the 13-th columns of $P$. The harmonic orders are marked by numbers.

antiresonance frequencies $\mathrm{A}$ and $\mathrm{B}$ of all modes of $H(s)$. The modes are numbered in the sequence of dominant harmonics 


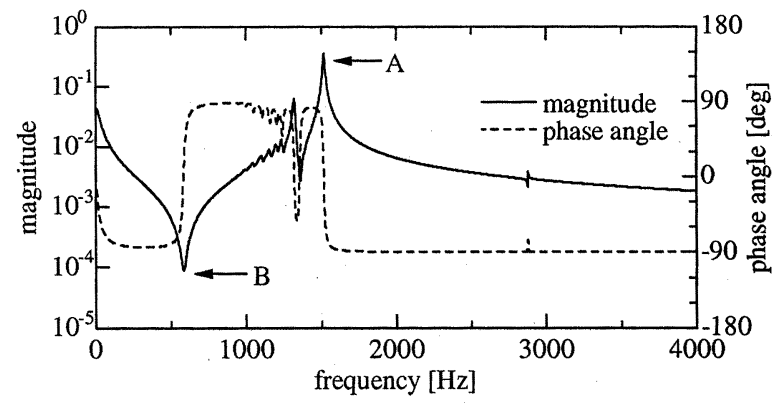

(a)

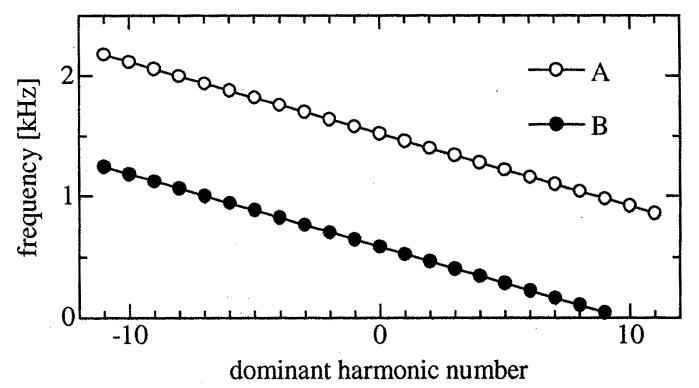

(b)

Fig. 13. (a) Frequency variation of eigenvectors carrying the information of 0 -th harmonic and (b) resonance and anti-resonance frequencies with respect to dominant harmonic numbers (A: resonance, B: antiresonance), for Test Network 2.

from -11 to 11 . The resonance and the antiresonance frequencies are both on descending straight lines with respect to the dominant harmonic number, and the distances in frequency between neighboring dominant harmonic numbers are exactly 60 Hz. This confirms that the curves in Fig. 11 consist of 23 congruent curves of Fig. 13(a) shifted by $-60 \mathrm{~Hz}$ with respect to the dominant harmonic number.

\section{CONCLUSIONS}

This paper presents the new concept of harmonic domain dynamic transfer function (HDDTF) and shows that the HDDTF can be used to characterize the dynamics of a nonlinear, time-periodic network seen from a port or multiple ports. The nonlinear state equations of the network are linearized about the periodic steady state, and the HDDTF is calculated by the linearized state equations with periodic coefficients. Thus, the HDDTF is a transfer-function matrix relating the vectors of harmonic domain input and output endowed with $s$-domain properties that give in the time domain the transient behavior superimposed on the steady state. Based on a modal decomposition analysis, it is shown that when the network exhibits half-wave symmetry, the odd and even harmonics are decoupled from each other and have their own modes. Two Test Networks are used to show their HDDTFs and the modal quantities. The results show that the dynamics of the networks are characterized in the form of the frequency responses of the HDDTF elements and the modal quantities. Possible resonances can be assessed by the frequency responses.
The next objective of this research is to use the HDDTF to develop a dynamic equivalent of a large-scale, nonlinear, time-periodic power network for off-line and real-time transient simulations. Exploiting the HDDTF information for power quality assessment is also an important application.

\section{REFERENCES}

[1] J. Arrillaga, N. R. Watson, and S. Chen, Power System Quality Assessment. New York: Wiley, 2000.

[2] R. C. Dugan, M. F. McGranaghan, and H. W. Beaty, Electrical Power System Quality. New York: McGraw-Hill, 1996.

[3] A. Semlyen, E. Acha, and J. Arrillaga, "Newton-type algorithms for the harmonic phasor analysis of nonlinear power circuits in periodical steady state with special reference to magnetic nonlinearities," IEEE Trans. Power Delivery, vol. 3, pp. 1090-1098, July 1988.

[4] E. Acha and M. Madrigal, Power System Harmonics-Computer Modeling and Analysis. New York: Wiley, 2001.

[5] T. Noda, A. Semlyen, and R. Iravani, "Reduced-order realization of a nonlinear power network using companion-form state equations with periodic coefficients," IEEE Trans. Power Delivery, vol. 18, pp. 1478-1488, Oct. 2003

[6] B. C. Smith, J. Arrillaga, A. R. Wood, and N. R. Watson, "A review of iterative harmonic analysis for AC-DC power systems," IEEE Trans. Power Delivery, vol. 13, pp. 180-185, Jan. 1998.

[7] R. A. Rohrer, Circuit Theory: An Introduction to the State Variable Approach. New York: McGraw-Hill, 1971.

[8] M. Farkas, Periodic Motions, Applied Mathematical Sciences. New York: Springer-Verlag, 1994, vol. 104.

[9] A. D'Angelo, Linear Time-Varying Systems: Analysis and Synthesis. Boston, MA: Allyn \& Bacon, 1970.

[10] A. Semlyen, "s-domain methodology for assessing the small signal stability of complex systems in nonsinusoidal steady state," IEEE Trans. Power Syst., vol. 14, pp. 132-137, Feb. 1999.

[11] MATLAB Function Reference (Volume 1: Language) Version 5, The Math Works, Inc., 1999.

Taku Noda (M'97) was born in Osaka, Japan, on July 4, 1969. He received the Bachelor's, Master's, and Ph.D. degrees in engineering from Doshisha University, Kyoto, Japan, in 1992, 1994, and 1997, respectively.

Currently, he is a Visiting Scientist at the University of Toronto, ON, Canada. He was with DEI Simulation Software, Neskowin, OR, in 1994, and a Consultant with Bonneville Power Administration, Portland, OR, in 1995. In 1997, he joined Central Research Institute of Electric Power Industry (CRIEPI), where he is Research Scientist. His main research interest is the transient analysis of power systems.

Dr. Noda is a member of IEE of Japan.

Adam Semlyen (LF'97) was born in 1923 in Rumania. He received the Dipl.Ing degree from the Polytechnic Institute of Timisoara, Rumania, in 1950, and the Ph.D. degree from the Polytechnic Institute of Iasi, Rumania, in 1965.

He began his career in Timisoara with an electric power utility and held academic positions at the Polytechnic Institute. In 1969, he joined the University of Toronto, ON, Canada, where he is a Professor in the Department of Electrical and Computer Engineering, emeritus since 1968. His research interests include steady state and dynamic analysis as well as computation of electromagnetic transients in power systems.

Reza Iravani (SM'00) received the B.Sc. degree in electrical engineering in 1976 from Tehran Polytechnique University, Tehran, Iran. He received the M.Sc. and $\mathrm{Ph} . \mathrm{D}$. degrees in electrical engineering from the University of Manitoba, Winnipeg, Canada, in 1981 and 1985 respectively.

Currently, he is a Professor at the University of Toronto, ON, Canada. His research interests include power electronics and power system dynamics and control. 